\title{
AN ITERATIVE SOLUTION TO THE EFFECTS OF CONCENTRATED LOADS APPLIED TO LONG RECTANGULAR BEAMS*
}

\author{
BY \\ C. A. M. GRAY \\ The University of Sydney
}

Summary. The analysis of the bending of thin deep rectangular beams by concentrated loads has already been treated by L.N.G. Filon and Th. v. Kármán. These authors consider an infinitely long beam and express the load as a Fourier integral, obtaining integral solutions for the stresses and displacements. In their integral form, these results are rather difficult to interpret, although F. Seewald, using Kármán's analysis, has calculated numerical values for the case of a single concentrated load.

In this paper, a totally different approach to the problem is made. The loaded area is conformally transformed to a circle, and, using Muschelisvili's transformation of the boundary conditions, the problem is solved in the plane of the circle. The solution is then obtained in the form of a complex power series. In this manner, a direct solution, to any required degree of accuracy, is readily obtained.

1. Theory. Consider a long deep rectangular beam resting on two supports placed at the same level, and loaded by a weight placed midway between them. The weight of the beam will be neglected, and the beam will be assumed to be in a state of generalised plane stress, the plane of the mean stress being that of the length and depth. To determine the local effects of the concentrated loads on the stresses and deflexion it is convenient to move the supports to infinity and treat the beam as an infinitely long strip of depth $2 h$ as is shown in Fig. 1. This procedure is that adopted by L.N.G. Filon, H. Lamb,

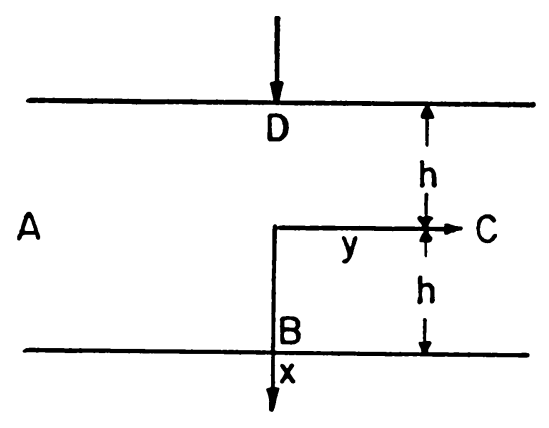

z-plane

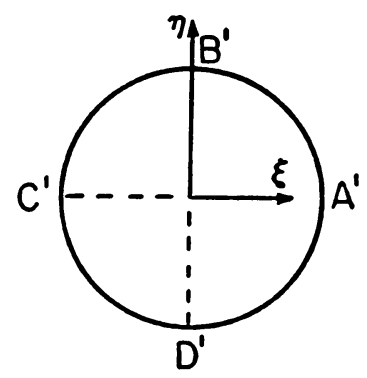

5-plane

FIG. 1.

and Th. v. Kármán $[1,2]$ in their solutions of the problem.

Transform the strip ABCD to the unit circle, $\gamma$, in the $\zeta$ plane by the transformation

$$
z=\frac{-2 i h}{\pi} \log \frac{1+\zeta}{1-\zeta}=-4 i h \pi^{-1}\left(\zeta+\zeta^{3} / 3+\zeta^{5} / 5+\cdots\right) .
$$

*Received May 23, 1952. 
Following Muschelisvili [3], define two functions $\Omega(z)$ and $w^{\prime}(z)$ of the complex variable $z$ by,

$$
\sigma_{x}+\sigma_{\nu}=R \Omega^{\prime}(z), \quad \sigma_{x}-\sigma_{y}+2 i \tau_{x y}=-\frac{1}{2}\left\{z \bar{\Omega}^{\prime \prime}(\bar{z})+\bar{w}^{\prime \prime}(\bar{z})\right\},
$$

where the dashes denote differentiation with regard to $z$ and the bars denote the conjugate function. Then, the boundary conditions in the $z$ plane can be expressed in the $\zeta$ plane by

where,

$$
\Omega[f(\sigma)]+f(\sigma) \bar{\Omega}_{z}^{\prime}(\bar{\sigma})+\bar{w}^{\prime}[f(\bar{\sigma})]=F_{1}+i F_{2},
$$

$$
z=f(\zeta)=\sum_{r=0}^{\alpha} u_{r} \zeta^{r}
$$

is the transformation relation, $\sigma$ is the value of $\zeta$ on the unit circle,

$$
\Omega_{z}^{\prime}(\zeta)=\frac{d}{d z}\{\Omega[f(\zeta)]\}
$$

$F_{1}+i F_{2}$ is the transform of $f_{1}+i f_{2}, f_{1}+i f_{2}=4 i \int_{0}^{s}\left(X_{v}+i Y_{v}\right) \mathrm{ds}, X_{v}, Y_{v}$, are the components of the boundary tractions, applied in the $z$ plane, and $s$ is the distance measured along the boundary in the $z$ plane.

Since $\Omega_{z}^{\prime}(\zeta)$ is analytic within the unit circle, we can express it by the Taylor's Series,

$$
\Omega_{z}^{\prime}(\zeta)=\sum_{r=0}^{r-\alpha} b_{r} \zeta^{r}
$$

Let us multiply both sides of (3) by $(1 / 2 \pi i) d \sigma /(\sigma-\zeta)$ and integrate around $\gamma$. Using Cauchy's Theorem and, neglecting the constant, we have,

$$
\Omega[f(\zeta)]=(1 / 2 \pi i) \int_{\gamma}\left(F_{1}+i F_{2}\right) d \sigma /(\sigma-\zeta)-(1 / 2 \pi i) \int_{\gamma} f(\sigma) \overline{\Omega_{2}^{\prime}}(\bar{\sigma}) d \sigma /(\sigma-\zeta)
$$

Similarly from the relation conjugate to (3) we obtain,

$$
w^{\prime}[f(\zeta)]=(1 / 2 \pi i) \int_{\gamma}\left(F_{1}-i F_{2}\right) d \sigma /(\sigma-\zeta)-(1 / 2 \pi i) \int_{\gamma} \bar{f}(\bar{\sigma}) \Omega_{2}^{\prime}(\sigma)^{-} d \sigma /(\sigma-\zeta) .
$$

Substituting (4) into (5), carrying out the integration and noting that

$$
\frac{d}{d \zeta} \frac{1}{2 \pi i} \int_{\gamma}\left(F_{1}+i F_{2}\right) \frac{d \sigma}{\sigma-\zeta}
$$

can be expressed as a power series, $\sum_{r=0} p_{r} \zeta^{r}$, we obtain the following infinite set of equations

$$
\sum_{0}^{n-\nu}(1+\nu) u_{\nu+1} b_{n-\nu}+(1+n) \sum_{\nu=0}^{\infty} u_{1+n+\nu} \bar{b}_{\nu}=p_{n} .
$$

Solving equation (7) gives $\Omega_{z}^{\prime}$. To complete the solution, we require $w^{\prime}(z)$ which is established in exactly the same way, using (6). The formula for $w^{\prime}(z)$ is then

$$
w^{\prime}(z)=(1 / 2 \pi i) \cdot \int_{\gamma}\left(F_{1}-i F_{2}\right) d \sigma /(\sigma-\zeta)-\sum_{r=0}^{\infty} c_{r} \zeta^{r},
$$

where the $c$ 's are given by the relations,

$$
c_{n}=\sum_{r=c} \bar{u}_{r} b_{n+r}
$$

A full and complete account of the derivation of (7) and (8) is given in Reference 4. 
2. Solution. Returning to the original problem, consider the given loading as a combination of the following two systems, where in system $\mathrm{B}$, the points $L, M, N, P$ are supposed to be very remote.
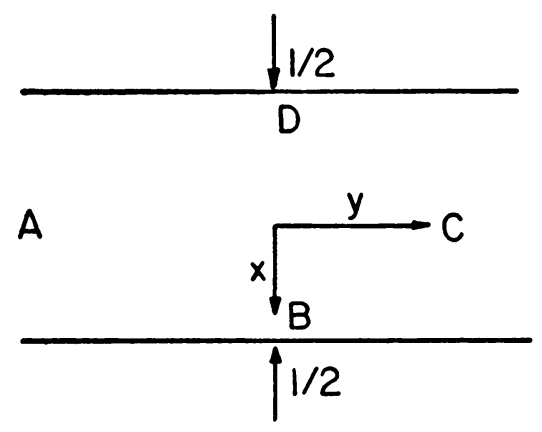

System A
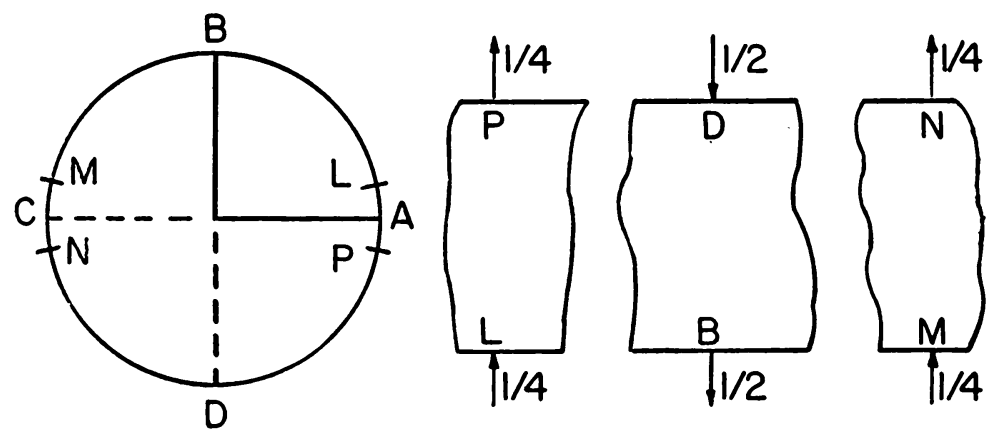

System B

Fig. 2.

Case A. Load function:

$$
\begin{aligned}
& \text { along } B C D, F_{1}+i F_{2}=-2 i, \\
& \text { along } D A B, F_{1}+i F_{2}=0 .
\end{aligned}
$$

Then,

$$
\begin{gathered}
(1 / 2 \pi i) \int_{\gamma}\left(F_{1}+i F_{2}\right) d \sigma /(\sigma-\zeta)=-\pi^{-1} \log \left(\sigma_{D}-\zeta\right) /\left(\sigma_{B}-\zeta\right) \\
\frac{d}{d \zeta}(1 / 2 \pi i) \int_{\gamma}\left(F_{1}+i F_{2}\right) d \sigma /(\sigma-\zeta)=\frac{2 i}{\pi}\left(1+\zeta^{2}\right)^{-1} \\
(1 / 2 \pi i) \int_{\gamma}\left(F_{1}-i F_{2}\right) d \sigma /(\sigma-\zeta)=\pi^{-1} \log \left(e^{i 3 \pi / 2}-\zeta\right) /\left(e^{i \pi / 2}-\zeta\right) .
\end{gathered}
$$


Equations (7) become

$$
\begin{aligned}
& 2 b_{0}+.33333 b_{2}+.2 b_{4}+.142857 b_{6}+.11111 b_{8}+\cdots=-(2 h)^{-1}, \\
& 2 b_{0}+1.6 b_{2}+.42857 b_{4}+.33333 b_{6}+.27273 b_{\mathrm{s}}+\cdots=(2 h)^{-1}, \\
& 2 b_{0}+1.7108 b_{2}+1.5556 b_{4}+.45455 b_{6}+.38462 b_{8}+\cdots=-(2 h)^{-1}, \\
& 2 b_{0}+1.778 b_{2}+1.6364 b_{4}+1.5385 b_{6}+.46666 b_{8}+\cdots=(2 h)^{-1} \text {, etc. }
\end{aligned}
$$

Using an iterative process to solve these equations we obtain for the 6 th and 7 th iterations the following values:

\begin{tabular}{|c|c|c|c|c|c|c|}
\hline Iteration & $b_{0} \times h$ & $b_{2} \times h$ & $b_{4} \times h$ & $b_{6} \times h$ & $b_{8} \times h$ & $b_{10} \times h$ \\
\hline 6 & -.32113 & .91461 & -1.0132 & .98457 & -.98724 & .98556 \\
\hline 7 & -.34898 & .88018 & -1.0381 & .97263 & -1.0143 & 1.0143 \\
\hline
\end{tabular}

From these we obtain

$$
\begin{gathered}
\Omega_{z}^{\prime}=-.329 / h+.895 \zeta^{2} / h-1.025 \zeta^{4} / h+.979 \zeta^{6} / h+\cdots, \\
=-\frac{1}{2 h} \frac{1-\zeta^{2}}{1+\zeta^{2}}+.171 / h-.105 \zeta^{2} / h-.025 \zeta^{4} / h+\cdots, \\
\Omega_{z}^{\prime \prime}=\frac{d \Omega^{\prime}}{d \zeta} \frac{d \zeta}{d z}=\frac{i \pi}{4 h^{2}} \zeta\left(1-\zeta^{2}\right)\left[\frac{2}{\left(1+\zeta^{2}\right)^{2}}-.21-.10 \zeta^{2}-.126 \zeta^{4}+\cdots\right] .
\end{gathered}
$$

Using these values in equations (8) and (9) we calculate $w^{\prime}(z)$ and $w^{\prime \prime}(z)$ as follows: $\dot{w}^{\prime}(z)=\frac{1}{\pi} \log \left(\frac{-i-\zeta}{i-\zeta}\right)-\frac{i \zeta}{2} \frac{1-\zeta^{2}}{1+\zeta^{2}}+\frac{2 i}{\pi}\left[-.55014 \zeta+.771667 \zeta^{3}\right.$

$$
\left.+.043 \zeta^{5}+\cdots\right]
$$

$w^{\prime \prime}(z)=\frac{1-\zeta^{2}}{h}\left[\frac{1.2854}{1+\zeta^{2}}-\frac{\pi}{2}\left[\frac{\zeta}{1+\zeta^{2}}\right]^{2}-.11763+.1075 \zeta^{2}+.107 \zeta^{4}+\cdots\right]$

Case B. Load function:

\begin{tabular}{|c|c|c|c|c|c|c|}
\hline For & $B M$ & $M C N$ & $N D$ & $D P$ & $P A L$ & $L B$ \\
\hline$F_{1}+i F_{2}$ & 0 & $-i$ & $-2 i$ & 0 & $-i$ & $-2 i$ \\
\hline
\end{tabular}

Then,

$$
\frac{1}{2 \pi i} \int_{\gamma}\left(F_{1}+i F_{2}\right) \frac{d \sigma}{\sigma-\zeta}=\frac{1}{2 \pi i}\left[-i \log \left(\frac{\sigma_{N}-\zeta}{\sigma_{M}-\zeta}\right)\left(\frac{\sigma_{D}-\zeta}{\sigma_{N}-\zeta}\right)\left(\frac{\sigma_{L}-\zeta}{\sigma_{P}-\zeta}\right)\left(\frac{\sigma_{P}-\zeta}{\sigma_{L}-\zeta}\right)^{2}\right]
$$

Now when $L$ and $P$ move to $A \sigma_{L}=\sigma_{P}=1$, and when $M$ and $N$ move to $C \sigma_{M}=\sigma_{N}=$ -1 . Thus,

$$
\frac{1}{2 \pi i} \int_{\gamma}\left(F_{1}+i F_{2}\right) \frac{d \sigma}{\sigma-\zeta}=\frac{-1}{\pi} \log \frac{-1-\zeta^{2}}{1-\zeta^{2}}
$$


The constants $b$ are purely imaginary and equations (7) become

$$
\begin{aligned}
b_{1}-2 b_{1} / 3-2 b_{3} / 5-2 b_{5} / 7-2 b_{7} / 9+\cdots & =-1 / h, \\
b_{1}+b_{3}-4 b_{1} / 5-4 b_{3} / 7-4 b_{5} / 9-4 b_{7} / 11+\cdots & =0, \\
b_{1}+b_{3}+b_{5}-6 b_{1} / 7-6 b_{3} / 9-6 b_{5} / 11-6 b_{7} / 13+\cdots & =-1 / h, \text { etc. }
\end{aligned}
$$

Assume

$$
\Omega_{z}^{\prime}=i \pi b_{1} z / 4 h+f_{3} \zeta^{3}+f_{5} \zeta^{5}+\cdots,
$$

i.e., assume that the $b$, are of the form

$$
\begin{aligned}
& b_{1}=b_{1}, \\
& b_{3}=b_{1} / 3+f_{3}, \\
& b_{5}=b_{1} / 5+f_{5}, \text { etc. }
\end{aligned}
$$

Then since

$$
\begin{gathered}
1-\frac{2}{3}-\frac{2}{5.3}-\frac{2}{7.5}-\frac{2}{7.9}+\cdots=0 \\
1+\frac{1}{3}-\frac{4}{1.5}-\frac{4}{3.7}-\frac{4}{5.9}+\cdots=0 \\
1+\frac{1}{3}+\frac{1}{5}-\frac{6}{1.7}-\frac{6}{3.9}-\frac{6}{5.11}+\cdots=0, \text { etc. }
\end{gathered}
$$

$b_{1}$ can be eliminated from the equations and we are left with the following system for the values of $f$ :

$$
\begin{aligned}
-.2 f_{3}-.142857 f_{5}-.111111 f_{7}-.090909 f_{9}+\cdots & =-1 / 2 h, \\
.107143 f_{3}-.111111 f_{5}-.090909 f_{7}-.076923 f_{9}+\cdots & =0, \\
.055556 f_{3}+.075758 f_{5}-.076923 f_{7}-.066667 f_{9}+\cdots & =-1 / 6 h, \text { etc. }
\end{aligned}
$$

To bring these into a form suitable for the iteration process subtract each equation from the one above it. Thus,

$$
\begin{aligned}
-.307143 f_{3}-.031746 f_{5}-.020202 f_{7}-.013986 f_{9}+\cdots & =-1 / 2 h, \\
.051587 f_{3}-.186869 f_{5}-.013986 f_{7}-.010256 f_{9}+\cdots & =1 / 6 h, \\
.021365 f_{3}-.027681 f_{5}-.135256 f_{7}-.007843 f_{9}+\cdots & =-1 / 6 h, \text { etc. }
\end{aligned}
$$

From these we obtain

\begin{tabular}{|c|c|c|c|}
\hline$f_{3} \div i / h$ & $1.581 \pm .004$ & $f_{9} \div i / h$ & $-.72 \pm .01$ \\
\hline$f_{5} \div i / h$ & $-.581 \pm .001$ & $f_{11} \div i / h$ & $1.25 \pm .01$ \\
\hline$f_{7} \div i / h$ & $1.33 \pm .02$ & $f_{13} \div i / h$ & $-.77 \pm .02$ \\
\hline
\end{tabular}


Thus

$$
h \Omega_{z}^{\prime}=i b_{1} z \pi / 4-i \zeta /\left(1+\zeta^{2}\right)+i \zeta\left(1+.581 \zeta^{2}+.419 \zeta^{4}+.33 \zeta^{6}+\cdots\right)
$$

and

$$
\begin{aligned}
h^{2} \Omega_{2}^{\prime \prime}=i b_{1} h \pi / 4+\frac{\pi}{4}\left(1-\zeta^{2}\right)^{2} /\left(1+\zeta^{2}\right)^{2}-\frac{\pi}{4}\left(1-\zeta^{2}\right)[1+ & 1.743 \zeta^{2} \\
& \left.+2.095 \zeta^{4}+\cdots\right]
\end{aligned}
$$

From formulae 8 and 9 and using the tabulated values of $f$, we have,

$$
\begin{aligned}
w^{\prime}(z)=i b_{1} z^{2} \pi / 8 h+\pi^{-1} \log \left(1+\zeta^{2}\right) /\left(1-\zeta^{2}\right)+i-\left(1+\zeta^{2}\right)^{-1} \\
\\
+(4 / \pi)\left[c_{0}+.972 \zeta^{2}+.749 \zeta^{4}+\cdots\right],
\end{aligned}
$$

and

$$
\begin{aligned}
h w^{\prime \prime}(z)=i \zeta /\left(1+\zeta^{2}\right)+(i \pi / 2) \zeta\left(1-\zeta^{2}\right) /\left(1+\zeta^{2}\right)^{2} & +i b_{1} z \pi / 4 \\
& +i \zeta\left(1-\zeta^{2}\right)\left(1.944+2.996 \zeta^{2}+\cdots\right) .
\end{aligned}
$$

The stress distribution for which $\Omega_{2}^{\prime \prime}=i b_{1} z \pi / 4 h, w^{\prime \prime}(z)=i b_{1} z \pi / 4 h$ is that due to pure flexure, provided $b_{1}=-i B_{1} / h$, where $B_{1}$ is real. From statics, we know that there is an infinite bending moment at the centre section due to the loads at infinity. But, from statics, at a section where the support loads are applied, the normal stress distribution must be equivalent to a moment of $h / 2 \pi$ as shown in Fig. 3. As this moment is applied

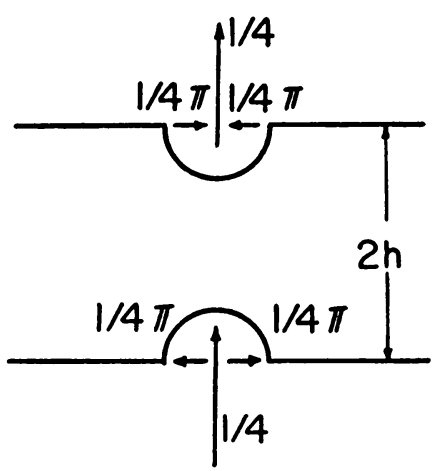

Fig. 3.

effectively at infinity, it must be included in the $b_{1}$ term. For as $\zeta \rightarrow \pm 1, R \Omega_{2}^{\prime} \rightarrow B_{1} z / 4 h^{2}$. Hence, $B_{1}$ is made up of two parts, that due to an infinite moment which will be represented by $b^{\prime}$ and that due to a moment of amount $h / 2 \pi$. Thus

$$
B_{1}=b^{\prime}+3 / \pi^{2}
$$

and

$$
\begin{array}{r}
h \Omega_{z}^{\prime}=b^{\prime} z \pi / 4 h+3 z / 4 \pi h-i \zeta /\left(1+\zeta^{2}\right)+i \zeta\left(1+.581 \zeta^{2}+.419 \zeta^{4}+\cdots\right), \\
w^{\prime}(z)=b^{\prime} z^{2} \pi / 8 h^{2}+3 z^{2} / 8 \pi h^{2}+\pi^{-1} \log \left(1+\zeta^{2}\right) /\left(1-\zeta^{2}\right)+i \\
-\left(1+\zeta^{2}\right)^{-1}+(4 / \pi)\left(c_{0}+.972 \zeta^{2}+\cdots\right)
\end{array}
$$


Equations $a$ to $f$ now give the solution to the problem. To calculate the stresses at any point we use equation (1) with the values of $\Omega_{z}^{\prime}, \Omega_{z}^{\prime \prime}$ and $w^{\prime \prime}$ obtained by substituting the corresponding values of $\zeta$ in the relevant equation $a$ to $j$. Fig. 4 gives the distribution

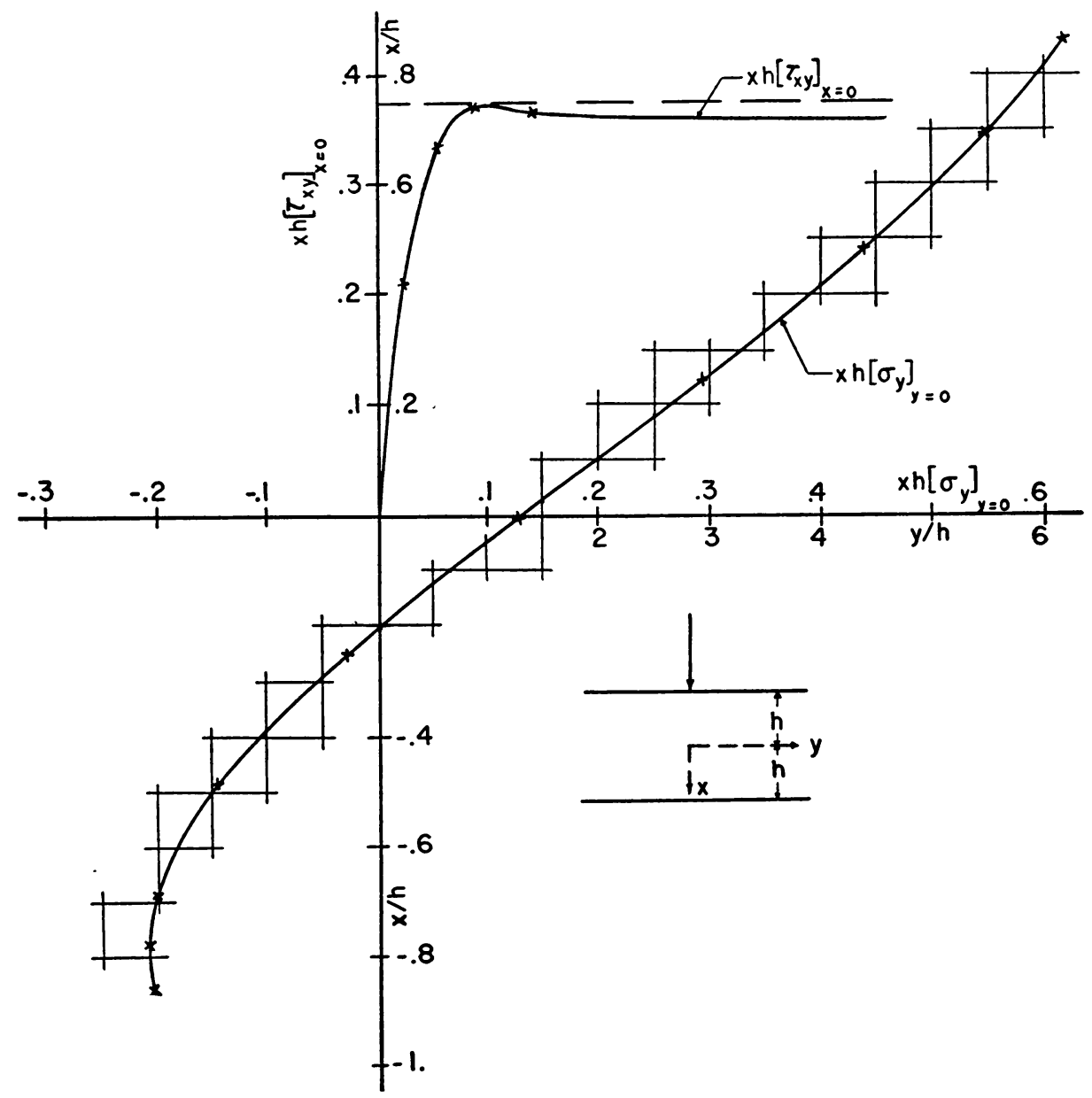

Fig. 4.

of normal stresses across $B D$ and the shear stress distribution along the $y$ axis.

The deflections $u$ and $v$ are readily obtained from formula (10)

$$
8 \mu(u+i v)=(3-\nu) /(1+\nu) \Omega(z)-z \bar{\Omega}^{\prime}(\bar{z})-\bar{w}^{\prime}(\bar{z}),
$$

where $\mu$ is the shear modulus; $\nu$ is Poisson's ratio, $u, v$ are the components of displacement in the $x$ and $y$ direction respectively; and $D$ is $u+i v$.

We are particularly concerned with the displacement and curvature of the $y$ axis and for this case some simplification of 10 is possible.

Differentiate both sides of (10) with regard to $y$, then,

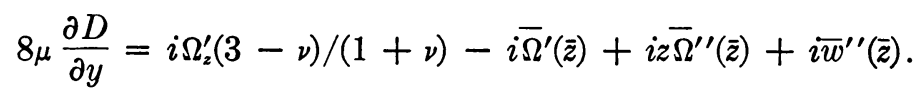


Since it is only the loading condition of case $B$ that will have any effect on the centre line we have for $x=0$,

$$
\begin{aligned}
& \zeta=\xi, \\
& i \Omega^{\prime}(z)=-i \bar{\Omega}^{\prime}(\bar{z}), \\
& i\left[z \bar{\Omega}^{\prime \prime}(\bar{z})+\bar{w}^{\prime \prime}(\bar{z})\right]=\frac{-\pi}{4 h^{2}} \cdot y \cdot\left(\frac{1-\xi^{2}}{1+\xi^{2}}\right)^{2}+\frac{1}{h} \cdot \frac{\xi}{1+\xi^{2}}+\frac{\pi}{2 h} \cdot \frac{\left(1-\xi^{2}\right)}{\left(1+\xi^{2}\right)^{2}} \xi \\
& +.944 \xi / h-.024 \xi^{2} / h+\cdots .
\end{aligned}
$$

and therefore

$$
\begin{aligned}
\left.8 \mu \frac{\partial u}{\partial y}\right]_{x=0}= & \frac{4}{1+\nu}\left[\frac{-\pi b^{\prime} y}{4 h^{2}}+\frac{1}{h} \cdot \frac{\xi}{1+\xi^{2}}-\left(.696 \xi+.480 \xi^{3}+\cdots\right)\right]-\frac{\pi}{4 h^{2}} \cdot y \\
& \cdot\left(\frac{1-\xi^{2}}{1+\xi^{2}}\right)^{2}+\frac{1}{h} \cdot \frac{\xi}{1+\xi^{2}}+\frac{\pi}{2 h} \cdot \xi \cdot \frac{1-\xi^{2}}{\left(1+\xi^{2}\right)^{2}}+(\xi / h)[.944-.024 \xi+\cdots] .
\end{aligned}
$$

To compare this result with the usual engineering form let $u_{1}$ be the deflection of $x=0$ and, considering the case $y>0$ let $\xi=-t$ and $E$ denote Young's Modulus. Then,

$$
\begin{aligned}
& \frac{d u_{1}}{d y}=\frac{\pi b^{\prime}}{4 h} \cdot \frac{y}{E h}+\frac{3}{8} \cdot\left(\frac{y}{h}\right)^{2} \frac{1}{E h}-\frac{1}{E h} \cdot \frac{t}{1+t^{2}}+\frac{1}{E h}\left[.696 t-.608 t^{2}\right. \\
&\left.+.480 t^{3}-.405 t^{4}+.358 t^{5}+\cdots\right]-\frac{1}{8 \mu h}\left[\frac{\pi}{4} \cdot \frac{y}{h} \cdot\left(\frac{1-t^{2}}{1+t^{2}}\right)^{2}+\frac{t}{1+t^{2}}\right. \\
&\left.\quad+\frac{\pi}{2} \cdot t \cdot \frac{1-t^{2}}{\left(1+t^{2}\right)^{2}}+.944 t-.024 t^{2}+\cdots\right]
\end{aligned}
$$

Using Euler's summation process we have for the first series on the right-hand side

$$
.696 t-.608 t^{2}+.480 t^{3}+\cdots=.696 \frac{t}{1+t}+.088\left(\frac{t}{1+t}\right)^{2}+\cdots .
$$

Then if $\Delta$ is the correction to the usual engineering deflection,

$$
\begin{aligned}
\frac{d \Delta}{d y}= & \frac{-1}{E h} \cdot \frac{t}{1+t^{2}}+\frac{1}{E h}\left(.696 \frac{t}{1+t}+.088\left(\frac{t}{1+t}\right)^{2}+\cdots\right) \\
& -\frac{1}{8 \mu h}\left[\frac{\pi}{4} \cdot \frac{y}{h}\left(\frac{1-t^{2}}{1+t^{2}}\right)^{2}+\frac{t}{1+t^{2}}+\frac{\pi}{2} \cdot t \cdot \frac{1-t^{2}}{\left(1+t^{2}\right)^{2}}+.944 t-.024 t^{2}+\cdots\right]
\end{aligned}
$$

Hence the correction to the usual engineering curvature is

$$
\begin{array}{r}
K=\frac{d^{2} \Delta}{d y^{2}}=\frac{\pi}{4 h^{2}} \cdot \frac{1-t^{2}}{E}\left(.696-1.216 \frac{t}{1+t}+.224\left(\frac{t}{1+t}\right)^{2}+\cdots\right) \\
-\frac{\pi}{4 h^{2} E}\left\{\frac{1-t^{2}}{1+t^{2}}\right\}^{2}-\frac{\pi}{32 \mu} \frac{1-t^{2}}{h^{2}} \cdot\left[.944+2 \frac{1-t^{2}}{\left(1+t^{2}\right)^{2}}-\frac{\pi y}{4 h} \cdot \frac{8 t\left(1-t^{2}\right)}{\left(1+t^{2}\right)^{3}}\right. \\
\left.+\frac{\pi}{2} \frac{1-6 t^{2}+t^{4}}{\left(1+t^{2}\right)^{3}}\right]
\end{array}
$$


and,

$$
\begin{aligned}
\Delta=\frac{-1}{\pi E} \log & \frac{1+t^{2}}{1-t^{2}}+\frac{1}{E}\left[.370 \frac{y}{h}+\frac{1.656}{\pi} \cdot \frac{1}{1+t}-\frac{.088}{\pi} \frac{1}{1+t^{2}}+\cdots\right] \\
& -\frac{1}{8 \mu} \frac{y}{h} \cdot \frac{t}{1+t^{2}}+\frac{1}{8 \mu} \cdot \frac{1}{1+t^{2}}+\frac{.236}{\pi \mu} \log \left(1-t^{2}\right)-\frac{1}{8 \mu}-\frac{1.568}{\pi E} .
\end{aligned}
$$

Figure 5 gives a plot of $K$ with $y$ assuming $\nu=0.3$ and shows that for $y>h, K$ is

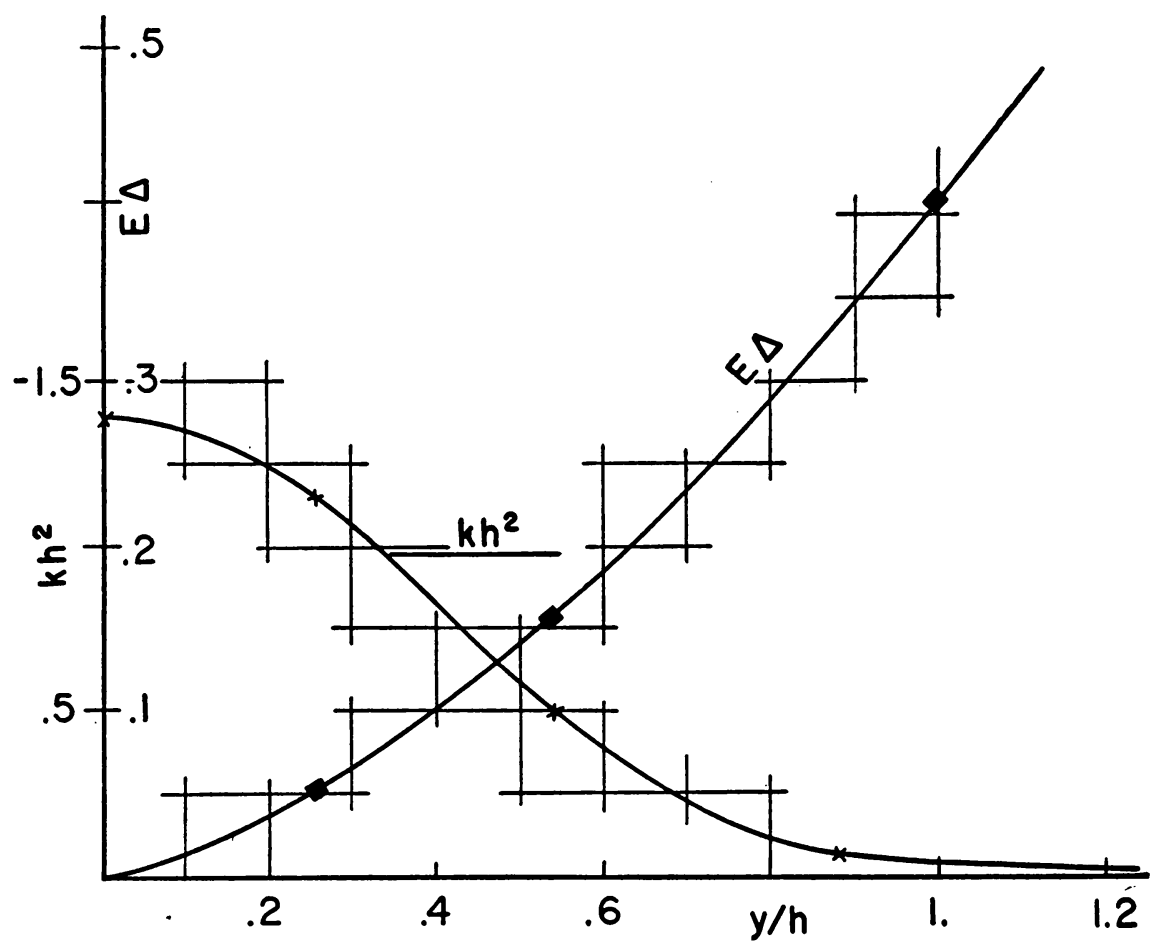

Fig. 5.

negligible. Hence the engineering approximation, that the curvature is proportional to the bending moment, is quite exact at a distance from the load greater than half the depth of the beam. Making the assumption that for $y>h$ the curvature of the centre line is proportional to the bending moment, we can then calculate the correction $\Delta$ which is also given in Fig. 5.

\section{REFERENCES}

1. L. N. G. Filon, Phil. Trans. (A) 201, 63 (1903).

2. Th. v. Kármán, see Trmoshenko, Theory of elasticity, p. 99-104.

3. N. Muschelisvili, Zeits. angew. Math. Mech. 13, 264 (1933).

4. C. A. M. Gray, Roy. Soc. N.S.W. Journal 85, 20 (1951). 